\title{
AVALIAÇÃO DOS EFEITOS DE UM PROTOCOLO DE EXERCICIOS COM BOLA SUÍÇA SOBRE A FUNCIONALIDADE DE INDIVIDUOSCOM DOENÇA DE PARKINSON
}

\author{
Isabella Morgado ROCHA ${ }^{1}$ \\ Wiviane Maria Torres de Matos FREITAS ${ }^{2}$ \\ ${ }^{1}$ Fisioterapeuta graduada pelo Centro Universitário do Pará - Belém (PA), Brasil.isabella_morgado@hotmail.com \\ ${ }^{2}$ Fisioterapeuta, Mestre em Doenças Tropicais e Docente do curso de fisioterapia no Centro Universitário do Pará - Belém \\ (PA), Brasil.wivianematos@yahoo.com.br
}

Recebido em: 23/04/2016 - Aprovado em: 21/11/2017 - Disponibilizado em: 30/12/2017

\begin{abstract}
RESUMO
Na Doença de Parkinson a principal manifestação clínica é a síndrome parkinsoniana, que pode se apresentar com rigidez, bradicinesia, tremor e/ou instabilidade postural. O objetivo do trabalho foi avaliar os efeitos de um protocolo de exercícios com bola suíça sobre a funcionalidade e equilíbrio em indivíduos com doença de Parkinson. Foram selecionados 5sujeitostriados a partir do Mini Exame do Estado Mental e escala de Estágios de Incapacidade de Hoehn e Yahr.A avaliação constituiu-se na aplicação daMedida de Independência Funcional e Escala de Equilíbrio de Berg. O protocolo fisioterapêutico de 10 sessões e duração de 50 minutos,formado por exercícios com uso da bola suíça visando trabalho de equilíbrio e funcionalidade por meio de movimentos frontal e lateral, apoio bipodal e unipodal, trabalho abdominal e de coordenação. Indivíduos com média de 61 anos de idade, de ambos os sexos, apresentaram melhora nas atividades de alcance frontal, apoio unipodal, transferências, no ato de vestir-se, entre outros. Não houve diferença estatisticamente significante no trabalho, entretanto pode-se inferir que o protocolo otimizou atividades funcionais e melhor equilíbrio e coordenação no desenvolvimento das mesmas para os indivíduos pesquisados. Outros estudos, com amostra maior, devem ser realizados para melhor analise do protocolo.
\end{abstract}

Palavras-chave: Doença de Parkinson.Fisioterapia.Equilíbrio.Exercício.

\section{EVALUATION OF THE EFFECTS OF EXERCISES WITH BALL SWITZERLAND PROTOCOL ON INDIVIDUALS FUNCTIONALITY WITH PARKINSON'S DISEASE}

\begin{abstract}
In Parkinson's disease, the major clinical manifestation is the parkinsonian syndrome, which can present with rigidity, bradykinesia, tremor and / or postural instability. The objective was to evaluate the effects of an exercise protocol with Swiss ball on the functionality and balance in individuals with Parkinson's disease. We selected 05 subjects screened from the Mini-Mental State Examination and scale of Stages of Hoehn and Yahr Disability. The evaluation consisted in the implementation of the Functional Independence Measure and Berg Balance Scale. The physiotherapeutic protocolof 10 sessions during 50 minutes consisted of exercises using the Swiss ball aimed at balancing work and functionality through front and side movements, bipedal and one-legged support, coordination and abdominal work. Individuals with an average of 61 years of age, of both sexes, showed improvement in frontal reach, one-legged support and transfers activities, in the act of dressing up, among others. There was no statistically significant difference at work, however it can be inferred that the optimized protocol functional activities, better balance and coordination on doing them for the individuals surveyed. Other studies with larger samples should be conducted to better analyze the protocol.
\end{abstract}

Keywords: Parkinson.Physiotherapy. Equilibrium. Exercise.

492

Revista da Universidade Vale do Rio Verde, Três Corações, v. 15, n. 2, p. 492-500, ago./dez. 2017 


\section{INTRODUÇÃO}

A Doença de Parkinson(DP) é uma afecção crônica e progressiva do sistema nervoso, caracterizada por sinais relacionados ao tônus muscular, execução dos movimentos, postura e marcha (PENTERNELLA E MARCON, 2009; MORREIRA ET AL, 2007). De uma maneira geral define-se com clareza a existência de uma perda neuronal progressiva no grupo de células ventro-laterais, da parte compacta da substância negra do mesencéfalo (SOUZA ET AL, 2011; SCALZO, 2011; PALERMO, 2009).

Na DP, a principal manifestação clínica é a síndrome parkinsoniana, que se apresenta com pelo menos dois dos quatro componentes básicos: rigidez, bradicinesia (acinesia), tremor e instabilidade postural. As alterações motoras encontradas nesses pacientes justificam o comprometimento na postura e equilíbrio interferindo diretamente nas atividades funcionais que são realizadas na postura ortostática (BARBOSA, SALLEM, 2005; GOULART, 2004).

Em virtude destas alterações no paciente com a doença de Parkinson, indica-se a associação do tratamento médico com o acompanhamento de profissionais como o fisioterapeuta e o fonoaudiólogo. Ressalta-se o uso da bola suíça dentre as condutas fisioterapêuticas, técnica desenvolvida na década de 70, com o intuito de ser um método para reabilitação de posturas anormais e problemas neurológicos (HAASE ET AL, 2008; QUINTELLA ET AL, 2008).

Para o portador da doença de Parkinson, condutas com o uso de bola suíça podeinfluenciar na estabilidade no paciente, por meio do treinamento da função de equilíbrio e mobilidade dos tecidos moles. Esta técnica também pode ser utilizada a fim de minimizar problemas motores do sujeito com Parkinson, em especial pelos agravos decorrentes da imobilidade que poderão assim interferir na funcionalidade de portadores da doença (HAASE ET AL, 2008; QUINTELLA ET AL, 2008).

Diante do exposto na literatura a fisioterapia poderá atuar na reeducação e manutenção do equilíbrio e da capacidade funcional do indivíduo com doença de Parkinson,todavia, novas estratégias ainda são pouco exploradas na literatura, entre elas a bola suíça. Por tal, este trabalho teve como objetivo avaliar os efeitos de um protocolo de exercícios com bola suíça sobre o equilíbrio e a funcionalidade de indivíduos com Doença de Parkinson.

\section{MATERIAIS E METODOS}

Tratou-se de um estudo experimental, controlado e não randomizado de caráter quantitativo, descritivo e analítico. Iniciado 
após aprovação do projeto pelo Comitê de Ética em Pesquisa do Centro Universitário do Pará(Parecer: 570.501) e aceite voluntário dos sujeitos, por meio da assinatura do Termo de Consentimento Livre e Esclarecido e o aceite da Instituição envolvida.

No que se refere aos critérios de inclusão, foram admitidos indivíduos adulto maior eidosos, com idadesa partir de 40 anos, até os 85 anos, de ambos os sexos, diagnosticados clinicamente com a doença de Parkinson e que apresentem acompanhamento médico regular. Idosos com o estado cognitivo preservado, avaliados através do Mini Exame do Estado Mental,e idosos classificados em estágio 1 (um) a 3(três) de acordo com a escala de Estágio de Incapacidade de Hoehn e Yahrmodificada, e que aceitassem participar voluntariamente da pesquisa.

Como critérios de exclusão adotou-se: idosos com mais de 85 anos, sujeitos que apresentassem alterações de comunicação que inviabilizem a pesquisa, indivíduos sem acompanhamento médico em virtude da interferência medicamentosa no tratamento, assim como aqueles que apresentassem alterações cardiovasculares sem o devido acompanhamento médico, também foram excluídos desse trabalho pacientes em estagio 4 (quatro) e 5 (cinco) de acordo com a escala de Estágios de Incapacidade de Hoehn e Yahr modificada ou os que não concordaram em participar espontaneamente da pesquisa.

Após triagem realizada por meio dos critérios acima descritos, foram selecionados cinco participantes paraavaliação através de fichas especificas, contendo variáveis como idade, sexo, tempo de diagnóstico da doença, história da doença atual e identificação das medicações em uso.

Posteriormente aplicou-se a MIF (Medida de Independência Funcional) para avaliar o nível de independência de cada paciente antes do início do plano terapêutico. Esta é composta por uma escala de 7 (sete) níveis que representam os graus de funcionalidade e avalia 18 (dezoito) funções. Cada uma das 18 funções é avaliada entre o escore de 1 e 7 a partir do qual é obtida uma média aritmética simples que permite classificar o nível de independência nas atividades de vida diária de cada paciente.

Para avaliação do equilíbrio foi usada a Escala de Equilíbrio de Berg (EEB). A escala tem uma pontuação máxima de 56 (cinquenta e seis) pontos, possuindo cada item uma escala ordinal de 5 (cinco) alternativas que variam de 0 (zero) a 4 (quatro) pontos.

O protocolo fisioterapêutico foi constituído por 10 exercícios pré-selecionados, todos com o uso da bola suíça, esses apresentavam variações em posições deitado, 
sentado e de pé, iniciando dos de menor complexidade para os de maior dificuldade.

Os exercícios do protocolo faziam uso da bola suíça visando trabalho de equilíbrio.Exercícios com variação de movimentos com alcance frontal e lateral (levar e trazer a bola para frente e para os lados) tanto em posições sentadas como ajoelhadas; Treino de equilíbrio sentado na bola com movimento de elevação do membro, de maneira alternada;Posição de pé e sentado na bola com simulações de tarefas que exigiam elevação de braços e rotações de tronco; Trabalho demusculatura abdominale músculos eretores da coluna na posição de ponte; Os dois últimos exercícios contavam com a contração isométrica da musculatura do membro inferior, além do treino especifico de agachamentos e de coordenação motora.

Após a realização de todas as intervenções e avaliações do estudo, as informações foram digitadas e tabuladas em banco de dados para a análise estatística dos mesmos. De acordo com a natureza das variáveis, realizou-se análise estatística descritiva, sendo informados os valores percentuais dos resultados obtidos, bem como os parâmetros de média, desvio padrão, mediana, mínimo e máximo. O banco de dados, bem como as tabelas e os gráficos foram construídos no
Microsoft EXCEL 2010. As análises foram executadas no software BioEstat 5.3.

\section{RESULTADOS}

Por meio dos dados obtidos na pesquisa, observou-se que dos 5 pacientes com DP que participaram na pesquisa,quatro eram do sexo masculino $80 \%$ e apenas 1 do sexo feminino $20 \%$, com idade média de $61 \pm 12,4$ anos de idade.

No que tange a aparição dos primeiros sintomas da doença, $40 \%$ dos pesquisados referiram acometimento de um hemicorpo, e $60 \%$ referiram comprometimento em ambos os lados.Quanto aos sintomas iniciais, 100\% dos pacientes apresentaram rigidez, bradicinesia e tremor, destaca-se que 20\% destes também apresentaram redução de força.

Para classificação do estágio da doença foi possível observar que a maioria, $80 \%$ dos indivíduos no estágio 3 e 20\% no estágio 2,5, de acordo com a escala de Hoehn e Yahr modificada conforme observa-se na Tabela I. 
Tabela I - Estatística descritiva do estágio da doença através da Escala de Hoehn e Yahr modificada. Pesquisa de campo, Belém-PA, 2014.

\begin{tabular}{lcc}
\hline Variáveis & $\mathbf{N}$ & Percentual \\
\hline Estágios da doença & & \\
0 & 0 & - \\
1,0 & 0 & - \\
1,5 & 0 & \\
2,0 & 0 & - \\
2,5 & 1 & $20 \%$ \\
3,0 & 4 & $80 \%$ \\
4,0 & 0 & - \\
5,0 & 0 & - \\
\hline
\end{tabular}

Com relação a MIF pode se observar que os pacientes avaliados no estudo antes e depois da aplicação do protocolo mostraram independência completa de acordo com a níveis de dependência funcional. (Tabela II).
Tabela II - Medida de independência funcional (MIF) antes e depois do protocolo terapêutico. Pesquisa de campo, Belém-PA, 2014.

\begin{tabular}{|c|c|c|c|c|c|c|}
\hline \multirow[b]{2}{*}{ Variáveis } & \multicolumn{3}{|c|}{ ANTES } & \multicolumn{3}{|c|}{ DEPOIS } \\
\hline & 䍃 & 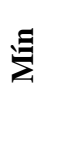 & 忘 & 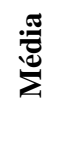 & $\Xi$ & 这 \\
\hline Alimentação & 7 & 7 & 7 & 7 & 7 & 7 \\
\hline HigienePessoal & 7 & 7 & 7 & 7 & 7 & 7 \\
\hline Banho (lavar o corpo) & 6,6 & 5 & 7 & 7 & 7 & 7 \\
\hline Vestirmetade superior & 6,8 & 6 & 7 & 6,8 & 6 & 7 \\
\hline Vestirmetade inferior & 7 & 7 & 7 & 7 & 7 & 7 \\
\hline Utilização do vasosanitário & 7 & 7 & 7 & 7 & 7 & 7 \\
\hline Controle da Urina & 7 & 7 & 7 & 7 & 7 & 7 \\
\hline Controle das Fezes & 7 & 7 & 7 & 7 & 7 & 7 \\
\hline & 55, & & & 55, & & \\
\hline Auto-Cuidados & 4 & 53 & 56 & 8 & 55 & 56 \\
\hline $\begin{array}{l}\text { Leito, cadeira, cadeira de } \\
\text { rodas }\end{array}$ & 7 & 7 & 7 & 7 & 7 & 7 \\
\hline Vasosanitário & 7 & 7 & 7 & 7 & 7 & 7 \\
\hline Banheira, chuveiro & 7 & 7 & 7 & 7 & 7 & 7 \\
\hline Mobilidade & 28 & 28 & 28 & 28 & 28 & 28 \\
\hline Compreensão & 7 & 7 & 7 & 7 & 7 & 7 \\
\hline Expressão & 7 & 7 & 7 & 7 & 7 & 7 \\
\hline Comunicação & 14 & 14 & 14 & 14 & 14 & 14 \\
\hline Interação Social & 7 & 7 & 7 & 7 & 7 & 7 \\
\hline Resolução de Problemas & 7 & 7 & 7 & 7 & 7 & 7 \\
\hline Memória & 7 & 7 & 7 & 7 & 7 & 7 \\
\hline \multirow[t]{2}{*}{ Cognição Social } & 21 & 21 & 21 & 21 & 21 & 21 \\
\hline & $\stackrel{+}{\infty}$ & $\stackrel{\emptyset}{=}$ & $\underline{2}$ & $\stackrel{\infty}{\infty}$ & $\stackrel{\infty}{=}$ & $\stackrel{\vartheta}{\partial}$ \\
\hline MIF TOTAL & & & & & & \\
\hline
\end{tabular}

Quanto a Escala de Equilíbrio de Berg pode se observar neste trabalho que os pacientes avaliados na pesquisa não apresentaram melhora estatisticamente significante antes e depois da aplicação do protocolo, como se observa na Tabela III. 
Tabela III - Escala de equilíbrio de BERG antes e depois do protocolo terapêutico. Pesquisa de campo, Belém-PA, 2014.

\begin{tabular}{|c|c|c|c|c|c|c|}
\hline \multirow[b]{2}{*}{ Variáveis } & \multicolumn{3}{|c|}{ ANTES } & \multicolumn{3}{|c|}{ DEPOIS } \\
\hline & 莺 & $\Xi$ & 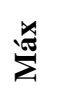 & 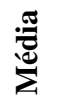 & $\sum$ & 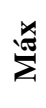 \\
\hline Sentado para empé & 4 & 4 & 4 & 4 & 4 & 4 \\
\hline Empésemapoio & 4 & 4 & 4 & 4 & 4 & 4 \\
\hline Sentadosemapoio & 4 & 4 & 4 & 4 & 4 & 4 \\
\hline Empé para sentado & 3,6 & 3 & 4 & 4 & 4 & 4 \\
\hline Transferências & 3,6 & 3 & 4 & 3,8 & 3 & 4 \\
\hline $\begin{array}{l}\text { Em pé com os olhos } \\
\text { fechados }\end{array}$ & 4 & 4 & 4 & 4 & 4 & 4 \\
\hline $\begin{array}{l}\text { Em pé com os pés } \\
\text { juntos }\end{array}$ & 4 & 4 & 4 & 4 & 4 & 4 \\
\hline $\begin{array}{l}\text { Reclinar à frente } \\
\text { com os braços } \\
\text { estendidos }\end{array}$ & 3,2 & 3 & 4 & 3,6 & 3 & 4 \\
\hline $\begin{array}{l}\text { Apanharobjeto do } \\
\text { chão }\end{array}$ & 4 & 4 & 4 & 4 & 4 & 4 \\
\hline $\begin{array}{l}\text { Virando-se para } \\
\text { olhar para trás }\end{array}$ & 4 & 4 & 4 & 4 & 4 & 4 \\
\hline Girando $360^{\circ}$ & 4 & 4 & 4 & 4 & 4 & 4 \\
\hline $\begin{array}{l}\text { Colocar os pés } \\
\text { alternadamente sobre } \\
\text { um banco }\end{array}$ & 3,6 & 3 & 4 & 3,6 & 3 & 4 \\
\hline $\begin{array}{l}\text { Em pé com um pé } \\
\text { em frente ao outro }\end{array}$ & 3 & 3 & 3 & 3,8 & 3 & 4 \\
\hline $\begin{array}{l}\text { Em pé apoiado em } \\
\text { um dos pés }\end{array}$ & 2,4 & 0 & 3 & 3,2 & 3 & 4 \\
\hline BERG TOTAL & 51,4 & 47 & 54 & 54 & 52 & 56 \\
\hline
\end{tabular}

\section{DISCUSSÃO}

Os pesquisados foram submetidos a 10 sessões de exercícios, e ressalta-se que a pesquisa encontrou dificuldades no tamanho amostral, entretanto os que se dispuseram foram assíduos aos atendimentos finalizando o estudo.

Foram submetidos ao trabalho em maioria homens com a DP,o que vai deencontro ao raciocínio de que a doença de Parkinson é dita como cosmopolita, pois não demonstra distinção de raça, cor ou condição social, mas tem uma predileção pelo sexo masculino sem uma explicação plausível (HAASE ET AL, 2008; QUINTELLA ET AL, 2008; CHRISTOFOLETTIET AL， 2010). Assim como o relato por Ferreira et al (2007)que vem trazendo estudos os quais comprovam uma maior prevalência da doença no sexo masculino, em uma razão aproximada de 3 homens para cada 2 mulheres.

O autor Silva et al.(2010) e Silva (2011) cita que a prevalência da DP aumenta com a idade, porém apresentariam uma média de acometimento mais frequente aos 55 a 65 anos de idade. Goulart (2004) concorda com a informação e trazem que apesar do acometimento em adultos na meia idade, os idosos têm se deparado comumente com a doença, estimando-se que $1 \%$ da população mundial de pessoas com mais de 65 anos 
apresentem tal distúrbio. Esta pesquisa limita-se ao tamanho amostral, porém vai de acordo com os achados dos autores, uma vez que indivíduos com média de 61 anos de idade apresentam a doença.

Ferreiraet al (2007) assim como Christofolettiet al (2010) trazem que o diagnósticoda doença, na sua maioria é feito após os 60 anos de idade, em virtude dos sintomas se manifestarem tardiamente ou discretamente.Neste trabalho apenas dois sujeitos foram diagnosticados após os 60 anos pelos mesmos motivos, a redução de força foi a primeira manifestação, não sendo relacionada precocemente a doença de Parkinson.

Em relação à evolução da sintomatologia, Barbosa e Sallem(2005)ressaltam que a DP e suas manifestações iniciam em um hemicorpo, o que ocorreu com a maioria dos sujeitos do grupo de estudo desta pesquisa, podendo passar progressivamente para o outro hemicorpo ou ambos. Neste estudo $60 \%$ dos pacientes relataramdurante a avaliação inicial que a doença manifestou-se primeiramente em um hemicorpo, progredindo posteriormente para ambos os lados do corpoe $40 \%$ relataram que a doença apareceu em ambos os hemicorpos.

Com a evolução dos tratamentos médicos foi necessária a criação de escalas para avaliação da DP. Os sujeitos desta pesquisa foram classificados quanto ao estágio da doença, em sua maioria encaixaram-se no estágio 3 (doença bilateral leve a moderada, alguma instabilidade postural, capacidade para viver independente). Visto a classificação neste estágio, poucas foram as alterações encontradas ao final do tratamento, porém identificou-se no presente estudo que houve melhora em alguns requisitos como estabilidade postural, transferências e os atos de vestir-se e banhar-se, proporcionando aos pesquisados aumentar sua independência e trouxe interferência em autoconfiança.

Apesar da classificação dos pacientes ser satisfatória para a independência dos mesmos, neste estudo observou-se que a maioria dos sujeitos apresenta o sintoma detremor em repouso e a redução de força, fatores estes que limitaram atividades instrumentais e laborais desenvolvidas por esses indivíduos. Houve até mesmo o afastamento de atividade ocupacional (fotógrafo) de um dos sujeitos em virtude dos sintomas decorrente da doença. Para este individuo, o protocolo pode otimizar a coordenação fina para a atividade de abotoarse, com a continuidade ao tratamento pode vislumbrar-se a retomada das atividades ocupacionais.

Para os pesquisadores deste trabalho, a funcionalidade destes pacientes melhorou em virtude do ganho alcançado na estabilidade postural. Verificou-se melhor desempenho em 
tarefas que necessitassem transferência da posição de pé para sentado, atividades de coordenação e equilíbrio como colocar os pés alternados sobre um banco, induzindo a maiores facilidades para locomoverem-se em transportes públicos e na rua, por exemplo.

Tais informações podem ser confrontadas com o estudo de Sant et al (2008), que retrata que pacientes com DP tendem ao sedentarismo, diminuição da força muscular e maior dependência funcional. Diante disso, os achados desta pesquisa se fazem importantes como possibilidade de diminuir os prejuízos da doença.

A literatura quanto trabalhos que envolvam a fisioterapia pelo uso da bola suíça em portadores da doença de Parkinson são escassos, pois a maioria visa estudo de flexibilidade, por este motivo a pesquisa apresenta limitações para maiores discussões das variáveis encontradas sobre funcionalidade e equilíbrio.

Vale ressaltar que com a evolução da DP, surgemcomplicações secundarias da doença decorrentes dos sinais e sintomas físicos contribuem para o comprometimento mental, emocional, social, físico e econômico o que vem ser extremamente incapacitante para o indivíduo, além de muitas vezes contribuirpara piora na qualidade de vida (LANA, 2007; QUINTELA ET AL, 2008; SILVA, CHRISTOFOLETTI ET AL, 2010; SILVA,
2012). Dos participantes da pesquisa, dois fazem uso de medicamentos antidepressivos pela DP, entretanto após o trabalho os pesquisados referiram melhor aceitação da doença, a partir da socialização proporcionadacom outros portadores da doença, e relataram melhora em alguns aspectos da sua qualidade de vida.

\section{CONCLUSÃO}

O trabalho conclui que após a aplicação do protocolo não se obteve resultado estatisticamente significante quanto as variáveis pesquisadas, todavia encontrou-se modificação na execução de algumas atividades.

Com isso, os efeitos observados foram melhora discreta na funcionalidade de banharse (MIF), e ganhos de equilíbrio estático e dinâmico para atividades de alcance frontal e lateral, apoio unipodal e bipodal com um pé a frente do outro, transferências e o ato de vestirse (EEB).

Neste estudo foram encontradas limitações quanto à literatura e quanto ao número da amostra, dificultando assim uma análise mais ampla dos possíveis resultados do protocolo aplicado. Sugere-se que novos estudos sejam realizados para melhor analise do protocolo, além da investigação de outros itens como a marcha e a percepção dos pacientes quanto à melhora psicossocial haja vista que os 
pacientes desse estudo relataram melhora nesses aspectos.

\section{REFERENCIAS}

BARBOSA, E.R.; SALLEM, F.A.S. Doença de Parkinson - diagnóstico. Revista

Neurociências.2005 v.13, n.3. p.158-165

CHRISTOFOLETTI, G; FREITAS, RT; CÂNDIDO, ER;CARDOSO, CS. Eficácia de tratamento fisioterapêutico no equilíbrio estático e dinâmico de pacientes com doença de Parkinson. Fisioterapia e Pesquisa, São Paulo, v.17, n.3, p.259-63, jul/set. 2010

FERREIRA, FV ; ZIEGLER, JR; STEIDL, SEM. Doença de Parkinson: Revisão bibliográfica. Disc. Scientia. Série: Ciências da Saúde, Santa Maria, 2007

GOULART, F. et al. Análise do desempenho funcional em pacientes portadores de doença de Parkinson. Acta Fisiátrica.2004;11(1):12-16

HAASE, D.C.B.V.; MACHADO, D.C.; OLIVEIRA, J.G.D. Atuação da Fisioterapia no paciente com doença de Parkinson. Fisioterapia e Movimento.2008;21(1):79-85

LANA, R. C. et al. Percepção da qualidade de vida de indivíduos com doença de Parkinson através do PDQ-39. Revista Brasileira de Fisioterapia. 2007

MORREIRA, CS; MARTINS, KFC; NERI, VC; ARAUJI, PG. Doença de parkinson: como diagnosticar e tratar. Revista Científica da FMC.2007 Vol. 2, nº 2.

PALERMO, S. et al. Avaliação e intervenção fonoaudiológica na doença de Parkinson: análise clínica-epidemiológica de 32 pacientes.
Revista Brasileira de Neurologia.2009; v.45, n.4. p.17-24.

PENTERNELLA FMN, MARCON SS.

Descobrindo a Doença de Parkinson: impacto para o parkinsoniano e seu familiar

Descobrindo a Doença de Parkinson: impacto para o parkinsoniano e seu familiar.

RevBrasEnfermagem.2009 62(1): 25-31

QUINTELLA RS, SCHATTI A, WIBELINGER LM, OLIVEIRA SG.

Qualidade de vida e funcionalidade na doença de Parkinson. RBCEH, Passo Fundo.2013, v. 10, n. 1, p. 104-112, jan./abril.paciente com doença de Parkinson. Fisioterapia e Movimento.2008;21(1):79-85

SANT, CR; OLIVEIRA, SG; ROSA, EL; DURANTE, JS; POSSER, SR. Abordagem fisioterapêutica na doença de Parkinson. Rev Brasileira Ciência Envelhecimento Humano 2008;5(1):80

SCALZO, P.L. et al. Impact of changes in balance and walking capacity on the quality of life in patients with Parkinson's disease. Arquivos de Neuropsiquiatria.2011 v.70, n.2. p.119.124.

SILVA, SS; PABIS, JVPC; ALENCAR, GA, SILVA, KB; PARTENELLA, FMN. Evolução da doença de Parkinson e comprometimento da qualidade de vida. RevNeurocienc2010 ; 18 (4): 463-468.

SILVA, CHD. Efeitos dos Exercícios Físicos sobre a Funcionalidade em Indivíduos Portadores da Doença de Parkinson.Faculdade de Santa Luzia. 2011

SOUZA CFM, ALMEIDA HCP, SOUSA JB, COSTA PH, SILVEIRA YSS, BEZERRA JCL. A Doença de Parkinson e o Processo de Envelhecimento Motor: Uma Revisão de Literatura. Rev. Neurociência 2011;19(4):71872. 